\title{
ANÁLISE RETROSPECTIVA DE 287 CASOS DE ABDOME AGUDO EM GINECOLOGIA E OBSTETRÍCIA
}

\author{
RETROSPECTIVE ANALYSIS OF 287 CASES OF ACUTE ABDOMEN \\ IN GYNECOLOGY AND OBSTETRICS
}

\author{
Eddie Fernando Candido Murta, ACBC-MG ${ }^{1}$ \\ Fabiana Sucupira Tiveron ${ }^{2}$ \\ Ana Cristina Macêdo Barcelos ${ }^{2}$ \\ Alessandra Manfrin ${ }^{2}$
}

\begin{abstract}
RESUMO: Objetivo: O abdome agudo em ginecologia e obstetrícia apresenta baixo risco de vida para a paciente, entretanto, o retardo no diagnóstico e tratamento influencia na morbi-mortalidade. O objetivo deste trabalho foi estudar as principais causas de abdome agudo em tocoginecologia. Métodos: Foram revisados 287 casos de abdome agudo em tocoginecologia de janeiro de 1987 a dezembro de 1997 atendidos na Disciplina de Ginecologia e Obstetrícia da Faculdade de Medicina do Triângulo Mineiro. Resultados: Os resultados mostraram que a prenhez ectópica foi a mais freqüente causa de abdome agudo hemorrágico com 98,5\% dos casos. Nestes casos, a dor pélvica foi o sintoma mais comum $(69,1 \%)$. Todas as pacientes foram submetidas à laparotomia e salpingectomia foi realizada em $92,6 \%$ dos casos. A causa mais freqüente de abdome agudo inflamatório foi a doença inflamatória pélvica com 94,8\%. A dor pélvica aguda estava presente em 91,5\% dos casos e a febre em 56,2\% casos. A penicilina foi usada com sucesso em 92,1\% dos casos. Do total de 201 casos de doença inflamatória pélvica, 13 (6,5\%) foram submetidos à laparotomia. Conclusões: Os autores concluem que o abdome agudo de causa tocoginecológica apresenta quadro clínico variável, portanto, o ginecologista deve estar atento para estabelecer diagnóstico e tratamento precisos.
\end{abstract}

Descritores: Abdome agudo; Gravidez ectópica; Doença inflamatória pélvica; Torção de anexo.

\section{INTRODUÇÃO}

O abdome agudo ginecológico é definido como uma dor abdominal repentina oriunda de uma afecção ginecológica que leva a paciente a procurar o médico ${ }^{1}$. É classificado em cirúrgico ou clínico. No abdome agudo ginecológico cirúrgico, a condição clínica da paciente impõe o procedimento cirúrgico de imediato. A prenhez ectópica rota é o exemplo mais freqüente ${ }^{2}$. O abdome agudo ginecológico clínico inclui as ginecopatias que não necessitam de tratamento cirúrgico imediato como a doença inflamatória pélvica (DIP) ${ }^{3}$. Pode ser classificado em hemorrágico e inflamatório. No primeiro, a prenhez ectópica também é a alteração mais freqüente e no segundo, a DIP ${ }^{1,4}$. A DIP ocorre principalmente nas mulheres jovens e nulíparas com a incidência de 850.000 casos/ ano nos $\mathrm{EUA}^{5,6}$. A prenhez ectópica ocorre entre 1:4.000 e 1:30.000 gestações ${ }^{2,7}$; a incidência nas pacientes submetidas a fertilização in vitro é de aproximadamente $1: 100^{8}$. Outras causas como a torção anexial e a ruptura de cisto anexial são mais $\operatorname{raras}^{9,10}$.

Na maioria das vezes as emergências em tocoginecologia são de baixo risco para as pacientes. Não obstante, a demora na indicação cirúrgica pode ser de alta morbidade ${ }^{3,11}$.

Poucos trabalhos na literatura nacional abordaram este tema. Portanto o objetivo deste trabalho é analisar retrospectivamente as principais causas de abdome agudo em ginecologia, verificando-se a sua freqüência, principais sinais e sintomas, métodos diagnósticos utilizados e a terapêutica empregada.

1. Professor Adjunto da Disciplina de Ginecologia e Obstetrícia.

2. Acadêmica de Medicina.

Recebido em 18/1/2000

Aceito para publicação em 12/9/2000

Trabalho realizado na Disciplina de Ginecologia e Obstetrícia da Faculdade de Medicina do Triângulo Mineiro. 


\section{MÉTODO}

O estudo foi realizado na Disciplina de Ginecologia e Obstetrícia, Departamento Materno-Infantil da Faculdade de Medicina do Triângulo Mineiro, Uberaba, MG. Foram analisados arquivos contendo registros de todos os pacientes adultos internados neste setor no período de janeiro de 1987 a dezembro de 1997. Destes foram excluídos casos com dados clínicos insuficientes ou casos onde os prontuários não foram encontrados, restando um total de 287 casos. Visando à padronização e à homogeneidade nos critérios diagnósticos, os procedimentos de coleta e registro de informações foram realizadas em conjunto por três observadoras treinadas. Foi utilizado um protocolo onde registrou-se a freqüência de diagnósticos de abdome agudo, detalhando-se algumas causas freqüentes, entre elas prenhez ectópica e doença inflamatória pélvica. Foram pesquisados idade, sintomas relatados pelo paciente durante a anamnese de internação, exame físico, realização ou não de ultra-som, hemograma, necessidade de transfusão sangüínea, tipo de tratamento realizado (clínico ou cirúrgico) e tempo de internação.

\section{RESULTADOS}

Foi analisado um total de 287 pacientes. Deste total, $75(26,1 \%)$ apresentavam abdome agudo do tipo hemorrágico. O diagnóstico mais freqüente foi prenhez ectópica com $68(90,7 \%)$ casos, seguido por cisto ovariano roto com sete $(9,3 \%)$ casos. Das restantes $212(73,9 \%)$ pacientes, $201(94,8 \%)$ apresentaram abdome agudo do tipo inflamatório, sendo que o diagnóstico mais freqüente foi a DIP com $201(94,8 \%)$ casos e a torção de anexo com 11 (5,2\%) casos.

A prenhez ectópica em 67 (98,5\%) casos foi diagnosticada como do tipo tubário, enquanto uma $(1,5 \%)$ foi prenhez ectópica ovariana. A média de idade foi de 26,9 $\pm 7,4$ anos. As manifestações clínicas estão descritas na Tabela 1, onde "outras" representam falha menstrual, palidez, sangramento vaginal, tenesmo e síncope. A ultrassonografia abdominal foi realizada em 45,6\% $(\mathrm{n}=31)$ das pacientes com prenhez ectópica. Níveis de hemoglobina menores do que $10 \mathrm{mg} / \mathrm{dl}$ estavam presentes em 32 (47\%) pacientes. Culdocentese foi realizada em 15 (22\%) pacientes, sendo que em 93,3\% (n=14) foi positiva. Em todos os casos o tratamento foi cirúrgico, tendo sido realizada salpingectomia em $98,5 \%$ das pacientes, e nas restantes associou-se ooforectomia. Em $56(82,4 \%)$ casos a incisão utilizada foi a de Pfannenstiel. Trinta e cinco $(51,4 \%)$ pacientes necessitaram de transfusão sangüínea durante a internação. O tempo médio de cirurgia foi de $126,9 \pm 23,4$ minutos. O tempo médio de internação foi $3,8 \pm 1,8$ dias.

As manifestações clínicas mais comuns da doença inflamatória pélvica estão na Tabela 2. A média de idade foi de 21,9 $\pm 8,3$ anos. Quatorze $(6,7 \%)$ pacientes no momento da internação, faziam uso de antibióticos. Níveis de hemoglobina menores do que $10 \mathrm{mg} / \mathrm{dl}$ foram encontrados em quatro $(0,02 \%)$ casos. O tratamento clínico foi realiza- do em $188(93,5 \%)$ casos. O tratamento cirúrgico foi realizado em $13(6,5 \%)$ pacientes (Tabela 3$)$. O tempo médio de cirurgia foi de178,8 $\pm 81,8$ minutos. O tempo médio de internação foi de $7,7 \pm 3$ dias.

Tabela 1

Manifestações clínicas da prenhez ectópica

\begin{tabular}{l|c|r}
\hline Manifestações clínicas & $N$ & $\%$ \\
\hline Dor hipogástrica & 47 & 69,1 \\
Dor abdominal difusa & 18 & 26,4 \\
Choque hipovolêmico & 17 & 25,6 \\
Dor epigástrica & 06 & 8,8 \\
Dor lombar & 04 & 5,8 \\
Dor escapular & 01 & 1,4 \\
Outras & 08 & 11,7 \\
\hline
\end{tabular}

Tabela 2

Manifestações clínicas da doença inflamatória pélvica

\begin{tabular}{l|c|c}
\hline Manifestações clínicas & $N$ & $\%$ \\
\hline Dor hipogástrica & 194 & 96,5 \\
Febre & 113 & 56,2 \\
Leucorréia & 82 & 40,8 \\
Disúria & 45 & 22,3 \\
Náuseas & 39 & 19,4 \\
Dor abdominal difusa & 38 & 18,9 \\
Dor lombar & 28 & 13,9 \\
Sangramento vaginal & 25 & 12,4 \\
Adinamia & 17 & 8,4 \\
Vômitos & 15 & 7,8 \\
Dor epigástrica & 13 & 6,4 \\
Cefaléia & 12 & 5,5 \\
Polaciúria & 11 & 5,4 \\
Dor flanco esquerdo & 07 & 3,5 \\
Diarréia & 06 & 03 \\
Dor flanco direito & 06 & 03 \\
Outros & 27 & 13,1 \\
\hline
\end{tabular}

Tabela 3

Operações realizadas no tratamento da doença inflamatória pélvica

\begin{tabular}{llc}
\hline Cirurgia & $N$ & $\%$ \\
\hline Salpingectomia & 06 & 46,2 \\
Anexectomia & 03 & 23,1 \\
Histerectomia e anexectomia & 04 & 30,7 \\
\hline Total & 13 & 100
\end{tabular}




\section{DISCUSSÃO}

A principal causa de abdome agudo hemorrágico em nosso serviço foi a prenhez ectópica. O quadro clínico é variável e, conseqüentemente, pode haver dificuldade no diagnóstico. A tríade clássica: dor, amenorréia e sangramento vaginal, acontece em cerca de $50 \%$ das pacientes, principalmente com prenhez ectópica rota ${ }^{2,10}$. Nossos achados demonstraram que a dor mais comum foi no hipogástrio. Segundo Tarazza \& Moore ${ }^{10}$, o diagnóstico da prenhez ectópica rota é fácil de ser feito quando existe o quadro de abdome agudo, choque hemorrágico e teste para gravidez positivo, entretanto, na maioria das vezes, o diagnóstico da prenhez ectópica não rota é mais difícil.

Os sinais clássicos de hemoperitôneo, como o sinal de Laffont, tenesmo e síncope podem ocorrer, entretanto não são comuns ${ }^{10}$. Na nossa casuística, o sinal de Laffont esteva presente em somente 1,4\% dos casos e amenorréia, sangramento vaginal, tenesmo, síncopes em 11,4\%. Somente $47 \%$ das pacientes apresentaram níveis de hemoglobina menores que $10 \mathrm{mg} \%$. Isto pode ser explicado pelo sangramento agudo, não havendo tempo para repercussões nos níveis de hemoglobina.

A culdocentese foi positiva em $93 \%$ dos casos. Embora, seja um exame traumático, acreditamos que possa contribuir nos casos em que há dúvida diagnóstica. Entretanto, há de se lembrar que a culdocentese positiva pode estar presente em somente $50 \%$ das mulheres com prenhez ectópica rota ${ }^{12}$.

A conduta cirúrgica na prenhez ectópica deve ser conservadora. A salpingectomia foi utilizada em nosso serviço em $92,6 \%$ das pacientes. Outra alternativa, como a anastomose terminoterminal, pode ser utilizadas em casos de desejo de procriação ou na ausência de trompa contralateral ${ }^{1}$. Apesar da urgência, a maioria das incisões foi a transversal, tipo Pfannenstiel. Usamos este tipo de incisão nas urgências por suspeita de prenhez ectópica por acreditarmos que o tempo de abertura da cavidade é rápido e a facilidade de acesso à pelve não é prejudicada. O tempo médio de cirurgia pode ser considerado longo em se tratando de operação simples, mas se deveu à grande quantidade de sangue e à exaustiva lavagem, da cavidade que realizamos de rotina nestes casos. O tempo de internação foi longo, principalmente, pela demora da paciente em recuperar-se do choque hipovolêmico que esteve presente em $25 \%$ dos casos e pela necessidade de transfusão sanguiínea realizada em mais de $50 \%$ das pacientes.

A DIP é mais freqüente em mulheres jovens e nulí$\operatorname{paras}^{4,13}$. As manifestações clínicas mais freqüentes foram a dor no hipogástro, febre e leucorréia. O diagnóstico da DIP em nosso serviço é baseado nos critérios de Hager ${ }^{14}$ que são a presença obrigatória de dor pélvica à mobilização do útero e anexos e a presença de pelo menos uma destas alterações: leucocitose $>10.000$ cels $/ \mathrm{mm}^{3}$, velocidade de hemossedimentação na primeira hora $>15 \mathrm{~mm}$, febre $\geq 38,5 \mathrm{C}^{\circ}$, leucorréia, suspeita de abscesso no ultra-som e/ ou no exame ginecológico. O critério de internação é baseado na presença de peritonite, ou abscesso ou nos casos em que há dúvida diagnóstica. O Center for Disease Control (CDC) preconiza que nas pacientes com DIP que têm indicação de internação, a associação de cefoxitina e doxiciclina ou clindamicina é a primeira escolha ${ }^{15}$. Entretanto, em nosso serviço o uso isolado de penicilina mostrou bons resultados, o que, aliado ao baixo custo torna este antibiótico ainda uma boa escolha para tratamento da DIP em paciente que não apresenta quadro de abscesso pélvico. A conduta cirúrgica foi rara, e nestes casos a salpingectomia foi o procedimento mais realizado. E este deve ser o tratamento de escolha, pois raramente há necessidade de histerectomia. Lembramos que todo o processo de infecção começa na trompa e a retirada deste órgão, resolve a maioria dos $\operatorname{casos}^{16,17}$.

A torção anexial é uma condição cirúrgica rara. Diamant $^{18}$ relata 300 casos descritos até 1971 . A maioria destes casos ocorreu na menacme e associados à gravidez, hidrossalpinge, cistos ovarianos ou paraovarianos e outras alterações anatômicas no anexo ${ }^{19}$. O sintoma mais freqüentes é a dor, que pode ser acompanhada de sangramento transvaginal, disúria e polaciúria ${ }^{20,21}$. Geralmente, o estado geral é bom, entretanto, a demora no diagnóstico e an terapêutica podem levar a paciente à peritonite grave e sep$\mathrm{se}^{22}$. O tratamento de escolha é a laparotomia ou laparoscopia e consiste na sua retirada nos casos de infecção e/ou necrose, e na conservação com fixação nos casos de boa vitalidade ${ }^{23}$.

\begin{abstract}
Background: The gynecology or obstetric acute abdomen in woman present a low risk, although the diagnosis and treatment may be a challenge. The aim of this study was to analize the most frequent causes of acute abdomen in gynecology and obstetrics. Methods: We reviewed 287 cases, from January 1987 to December 1997, attending in Discipline of Gynecology and Obstetrics of the Faculty of Medicine of "Triângulo Mineiro". Results: Ouur results showed that ectopic pregnancy was the most frequent cause of hemorrhagic acute abdomen, with $98.5 \%$ of the cases. Pelvic pain was the most common symptom (69.1\%). All patients were submitted to a laparotomy; salpingectomy was performed in $92.6 \%$ of cases. The most frequent cause of inflammatory acute abdomen was inflammatory pelvic disease, with $92.6 \%$. Acute pelvic pain was present in $96,5 \%$ of the cases and fever in $56.2 \%$. Penicillin was successfully used in 92.1\%. From the 206 cases of IPD, 13(6.5\%) were submitted to laparotomy. Conclusions: The authors concluded that acute abdomen for ginecology or obstetrical disorders shows a variability of symptoms that may lead to a wrong diagnostic. Meanwhile, the gynecologist must be aware of these variabilities to perform a correct diagnosis.
\end{abstract}

Key Words: Acute abdomen; Ectopic pregnancy; Inflammatory pelvic disease; Torsion of the adnexa 


\section{REFERÊNCIAS}

1. Oliveira LJ, Urbanetz AA, Nascimento RMAM. Abdome Agudo em Ginecologia. In:Halbe HW ed. Tratado de ginecologia. São Paulo. $2^{\circ}$ edição .Editora Roca Ltda 1995.

2. Emerson DS, McCord ML. Clinican's approach to ectopic pregnancy. Clin Obstet Gynecol, 1996; 39(1): 199222.

3. Rezende WW, Rezende CS, Pinotti M et al. Urgências em ginecologia: abdome agudo. RBM-Ginecologia e Obstetrícia, 1996; 6: 353-358.

4. Pires RA, Bóscollo ACP, Murta EFC. Abdome agudo em ginecologia. J Bras Ginecol, 1997; 107(10): 343-346.

5. Washington AE, Aral SO, Wolner-Hansen P et al. Assessing risk for pelvic Inflammatory disease and its sequel. JAMA, 1991; 226:2581-2586.

6. Curran JW, Rendtorff RC, Chandler RW et al. Female gonorrhea: Its relation to abnormal uterine bleeding urinary tract symptoms, and cervicitis. Obstet Gynecol, 1975;45: 195-198.

7. DeVoe RW, Pratt JH. Simultaneous intrauterine and extrauterine pregnancy. Am J Obstet Gynecol, 1948;56: 1119-1126.

8. Rizk B, Tan SL, Marcos S et al. Heterotopic pregnancies after in vitro ertilization and embryo transfer. Am J Obstet Gynecol, 1991;164: 161-164.

9. Murta EFC, Paschoini MC, Silva SR. Torção isolada da trompa de Falópio em uma paciente virgem: uma rara condição cirúrgica. J Bras Ginecol, 1994;104(9): 327-330.

10. Tarazza HM, Moore RD. Gynecoloc causes of the acute abdomen and the acute abdomen in pregnancy. Surg Clin North Am, 1997;77(6): 1371-1395.

11. El-Amin Ali M, Yahia Al-Shehrin M, Zaki ZMS et al. Acute abdomen in pregnancy. International Federation Gynecology and Obstetrics, 1998; 62: 31-36.

12. Vermesh M, Graczkowski JW, Saver MV. Reevaluation of the role of culdocentesis in the management of ectopic pregnancy. Am J Obstet Gynecol, 1990;162: 411.

13. Aral SO, Mosher WD, Cates W. Self-reported pelvic inflammatory disease in the United States 1988. JAMA, $1991 ; 266: 2570-3$.

14. Hager WD, Eschenbach DA, Spence MR. Criteria for diagnosis and grading of salpingitis. Obstet Gynecol, 1983; 61: 113-116.
15. Centers for disease control: pelvic inflammatory disease: guidelines for prevention and management. MMWR, 1993;42: 75-83.

16. Ginsberg DS, Stern JL, Hamod KA et al. Tubo-ovarian abscess, a retrospective review. Am J Obstet Gynecol, 1980;138: 1055.

17. Rivlin ME, Hunt JA. Ruptured tubo-ovarian abscess: Is hysterectomy necessary? Obstet Gynecol, 1977;50:518.

18. Diamant YZ. Torsion of hydrosalpinx: report of four cases. Int Surg, 1972;57: 303-306.

19. Provost RW. Torsion of the normal fallopian tube. Obstet Gynecol, 1972;1: 80-82.

20. Barnes WS, Schantz JC, Shochat SJ. Torsion of the fallopian tube in a premenarcheal patient. Am J Dis Child, 1977;131: 1297-1298.

21. Best CL, Feldman DB, Sobenes JR et al. Unexplained displacement of ypsilateral ovary and fallopian tube. Obstet Gynecol, 1991; 78: 558-560.

22. Nichols DH, Julian PJ. Torsion of the adnexa to cavanagh D, ed. Gynecologic Emergencies. Clinical Obstetrics and Gynecology. New York, NY, Harper \& Row, 1985: 375-380.

23. Shalev E, Mann S, Romano S et al. Laparoscopic detorsion of adnexa in childhood: a case report. J Pediatr Surg, 1990; 26: 1193-1194.

Endereço para correspondência:

Prof. Eddie Fernando Candido Murta

Rua Getúlio Guaritá, s/no - Abadia

38025-440 - Uberaba-MG

E-mail:eddiemurta@mednet.com.br 